\title{
Review: postmenopausal hormone replacement therapy is associated with a decreased risk of colorectal cancer
}

Grodstein F, Newcomb PA, Stampfer MJ. Postmenopausal hormone therapy and the risk of colorectal cancer: a review and meta-analysis. Am J Med 1999 May;106:574-82.

QUESTION: Is postmenopausal hormone replacement therapy associated with a decreased risk of colorectal cancer in women?

\section{Data sources}

English language studies were identified by searching Medline (1966 to September 1998) using the subject headings for colorectal, colon, and rectal neoplasms or the textwords "colorectal, colon, and rectal cancer" and the subject headings "estrogen, estrogen replacement therapy" or the textwords "hormone replacement therapy, postmenopausal hormones, or non contraceptive hormones". Bibliographies were also reviewed.

\section{Study selection}

Studies were selected if they provided estimates of the risk of colon or rectal cancer in relation to postmenopausal hormone use.

\section{Data extraction}

Data were extracted on study design, cases of colon cancer, categories of hormone use (ever use, current use, 95\% confidence intervals (CIs).

\section{Main results}

18 studies (9 prospective, 8 case control, and 1 clinical trial) were included in the analysis. The weighted average of estimated RRs was calculated by giving each study a weight proportional to its precision (the inverse of its variance). Larger studies (with more precise estimates and narrower confidence limits) were given greater weight than smaller studies. Meta-analyses were done using a fixed effects model. Women who had ever used postmenopausal hormones had a lower risk of colon cancer and a lower risk of rectal cancer than women who had never used postmenopausal hormones; analyses excluding studies that included premenopausal women as part of the "never used" group showed similar results (table). Analysis of 10 studies that reported on the timing of hormone use showed that current hormone use was associated with a lower risk of colorectal cancer than never use (RR 0.66, CI 0.59 to 0.74). Both short and long durations of current use were associated with a decreased risk of colorectal cancer

Age-adjusted relative risk $(R R)$ of colon and rectal cancer for women who had ever used postmenopausal hormones compared with those who had never used hormones

Source of funding: National Institutes of Health.

For correspondence: $\mathrm{Dr} F$ Grodstein, Channing Laboratory, 181

Longwood Avenue Boston, MA 02115

USA.Fax +1617525 2008.

\begin{tabular}{clll} 
Colon & 18 & $0.80(0.74$ to 0.86$)$ & $\mathrm{p}=0.02 \dagger$ \\
\cline { 2 - 4 } & $13^{\star}$ & $0.76(0.70$ to 0.82$)$ & $\mathrm{p}=0.32$ \\
\hline Rectal & 10 & $0.81(0.72$ to 0.92$)$ & $\mathrm{p}=0.03 \dagger$ \\
\cline { 2 - 4 } & $7^{\star}$ & 0.81 (CI not available) & $\mathrm{p}=0.06 \dagger$
\end{tabular}

"Excluding studies that included premenopausal women in the "never used" group. †Significant heterogeneity existed among study results. and duration of use), odds ratios, relative risks (RRs), and

when compared with never use (5 studies, RR 0.61, CI 0.48 to 0.79 for short duration and RR 0.67 , CI 0.56 to 0.79 for long duration).

\section{Conclusion}

Postmenopausal hormone use is associated with a decreased risk of colon cancer and rectal cancer in women.

\section{COMMENTARY}

Colorectal cancer is one of the more common cancers and accounts for many cancer deaths. ${ }^{1}$ In their review of predominantly observational studies, Grodstein $e t$ al compared women who had ever used postmenopausal hormones with women who had never used them and found a $20 \%$ reduction in cancer of the colon and a $19 \%$ reduction in cancer of the rectum. The greatest protection was seen in women currently taking postmenopausal hormones and it decreased substantially after cessation of hormone use. The authors also provided evidence about the biological basis for this association.

This review consolidates the results of 18 studies, only 1 of which is a randomised controlled trial. The authors have identified the design of each study and have weighted the studies in the meta-analysis according to study size. They did not, however, assess the methodological quality of the studies with respect to important criteria such as blinding of data collectors to exposure (ie, hormone use) or to outcome (ie, colon or rectal cancer) depending on the study design and completeness of follow up of study participants in the prospective studies. They limited their search to only 1 database (Medline) and did not include unpublished studies. The authors provided limited data on type of hormone use but note that only 3 studies examined the effect of combined oestrogen and progestin use. For these reasons, validation of the findings of this review by future reviews on the same topic will be important to increase confidence in the results.

In the meantime, how can this information be used by clinicians? Postmenopausal women facing the decision of whether to take hormone replacement therapy must be informed about all the potential risks and benefits. This review provides preliminary evidence of a decreased risk of colorectal cancer among women who take postmenopausal hormones.

Dinah Gould, RGN, RNT, PhD Professor of Nursing, Faculty of Health South Bank University London, UK

Alba DiCenso, RN, PhD Professor, School of Nursing McMaster University Hamilton, Ontario, Canada 